\title{
ASYMPTOTIC HOMOGENISATION IN STRENGTH AND FATIGUE DURABILITY ANALYSIS OF COMPOSITES
}

\author{
S. E. Mikhailov \\ Div. of Mathematics, Glasgow Caledonian Univ., Glasgow, G4 OBA, UK \\ s.mikhailov@gcal.ac.uk
}

J. Orlik

Fraunhofer Institut für Techno- und Wirtschaftsmathematik

Gottlieb-Daimler-Str. 49, D-67663 Kaiserslautern, Germany

orlik@itwm.fhg.de

Abstract Asymptotic homogenisation technique and two-scale convergence is used for analysis of macro-strength and fatigue durability of composites with a periodic structure under cyclic loading. The linear damage accumulation rule is employed in the phenomenological micro-durability conditions (for each component of the composite) under varying cyclic loading. Both local and non-local strength and durability conditions are analysed. The strong convergence of the strength as the structure period tends to zero is proved and its limiting value is estimated.

\section{INTRODUCTION}

Different homogenisation methods are widely used for obtaining homogenised macro-stress fields and effective elastic properties of composites. In $[2,6]$, the first approximation to the micro-stress field was derived from the properties of the components, micro-geometry of the composite and the applied macro-loads. Convergence of the micro-stresses to some limit, as the structure period tends to zero, can be proved by the twoscale homogenisation technique $[1,3]$. The present paper is based on the fact that this limit is exactly the first term of the micro-stress field approximation, which is the product of the homogenised stress tensor, depending only on macro-geometry and boundary conditions, and the so-called stress concentration tensor, related only to the micro-geometry and stiffness tensors of composite components. 
Let $B$ be a Banach space and $\tilde{\Omega}$ be an open or closed domain in a finite-dimensional space. Then $C(\tilde{\Omega}, B)$ denotes the space of continuous Banach-valued functions $f: \tilde{\Omega} \ni x \mapsto f(x) \in B$, that is such that $\left\|f\left(x_{1}\right)-f\left(x_{2}\right)\right\|_{B} \rightarrow 0$ as $\tilde{\Omega} \ni x_{2} \rightarrow x_{1}$ for any $x_{1} \in \tilde{\Omega}$. Let $\mathcal{M}(\tilde{\Omega})$ be the space of all bounded functions on $\tilde{\Omega}$.

\section{ELEMENTS OF STRENGTH ANALYSIS}

For a bounded stress field $\sigma_{i j}(y), i, j=1, \ldots, 3$, any local strength condition for micro-stresses at a point $y \in \Omega \subset \mathbb{R}^{k}(k$ is 1,2 or 3 ) can be written in the form $\Lambda(\sigma(y), y)<1$, where $\Lambda \in C\left(\mathbb{R}^{3 \times 3}, \mathcal{M}(\Omega)\right)$ is a normalised equivalent stress function, a material characteristic, which is non-negative and positively homogeneous of the order +1 w.r.t. $\sigma$.

Example 1. For some materials $\Lambda$ can be associated with the von Mises equivalent stress, $\Lambda_{M}(\sigma(y), y)=\left\{\left[\left(\sigma_{1}(y)-\sigma_{2}(y)\right)^{2}+\left(\sigma_{2}(y)-\sigma_{3}(y)\right)^{2}+\right.\right.$ $\left.\left.\left(\sigma_{3}(y)-\sigma_{1}(y)\right)^{2}\right]\right\}^{1 / 2} /\left(\sqrt{2} \sigma_{r}(y)\right)$, or with the Tresca equivalent stress, $\Lambda_{T}(\sigma(y), y)=\max _{k, m}\left|\sigma_{k}(y)-\sigma_{m}(y)\right| / \sigma_{r}(y)$, where $\sigma_{1}, \sigma_{2}, \sigma_{3}$ are the principal stresses and $\sigma_{r}$ is a known uniaxial strength of material.

Assuming body rupture means rupture of at least one of its points, the (initial) local strength condition for the whole body is then

$$
\sup _{y \in \Omega} \Lambda(\sigma(y), y)<1 .
$$

Such local strength condition, however, is generally not applicable to unbounded stress fields since the conditions will predict fracture under virtually any singular stress field.

For more general, especially singular stress fields, e.g. belonging to $L_{2}(\Omega)$, a (point) non-local strength condition $\Lambda^{\odot}(\sigma ; y)<1$ can be applied. Here $\Lambda^{\odot}(\sigma ; y)$ is a normalised equivalent stress functional, which is defined on the tensor-functions $\sigma_{i j} \in L_{2}(\Omega)$ and is non-negative positively homogeneous of the order +1 w.r.t. $\sigma$, see [5].

Particularly for some materials $\Lambda^{\odot}$ can be related with a weighted averaging of $\sigma_{i j}(x), x \in \Omega$ along some neighbourhood of the point $y$,

$$
\Lambda^{\odot}(\sigma ; y):=\Lambda\left(\sigma^{\odot}(y), y\right), \quad \sigma_{i j}^{\odot}(y):=\int_{\Omega} w_{i j k l}(y, x) \sigma_{k l}(x) d x,
$$

where $\sigma_{i j}^{\odot} \in C(\bar{\Omega})$ is an auxiliary non-local stress tensor, and the weight $w \in C\left(\bar{\Omega}, L^{2}(\Omega)\right)$ is a material characteristics, such as $\int_{\Omega} w_{i j k l}(y, x) d x=$ $\delta_{i j} \delta_{k l}$. Then the non-local strength condition for the whole body is $\sup _{y \in \Omega} \Lambda\left(\sigma^{\odot}, y\right)<1$. 
Example 2. (i) If $w_{i j k l}(y, x)=\delta_{i j} \delta_{k l}\left\{\begin{array}{ll}\frac{3}{4 \pi d^{3}}, & |x-y|<d \\ 0, & |x-y| \geq d\end{array}\right.$ for $3 D$, where $d$ is a material constant, then $\sigma_{i j}^{\odot}(y)=\frac{3}{4 \pi d^{3}} \int_{|x-y|<d} \sigma_{i j}(x) d x$.

(ii) If $w_{i j k l}(y, x)=\delta(x-y) \delta_{i j} \delta_{k l}$, where $\delta(x-y)$ is the Dirac function, then $\sigma_{i j}^{\odot}(y)=\sigma_{i j}(y)$, and the non-local strength-condition coincides with the local one.

\section{ELEMENTS OF FATIGUE DURABILITY ANALYSIS}

Pure fatigue under cyclic loading is characterised by dependence of the durability on the loading history considered as a sequence of loading events but not on time or rate of loading. Then the cycle number $n$ can be considered as a a discrete or continuous time-like parameter, more relevant to fatigue than the natural continuous time $t$. The Wöhler $\mathrm{S}-\mathrm{N}$ durability diagram (Wöhler function) for a material under an uniaxial regular periodic cycling with constant stress range $\Delta \sigma=\sigma_{\max }-\sigma_{\min }$ and mean stress $\sigma_{m}=\left(\sigma_{\max }+\sigma_{\min }\right) / 2$, is a dependence of the critical number of cycles $n^{*}\left(\Delta \sigma, \sigma_{m}\right)$ to rapture, e.g. on $\Delta \sigma$. For a multiaxial in-phase periodic cycling, we consider $\Delta \sigma=\Delta \sigma_{i j}$ and $\sigma_{m}=\sigma_{m i j}(i, j=1,2,3)$ as tensors. For simplicity, suppose further that $\sigma_{m i j}=0$ and $1 / n^{*}(\Delta \sigma)$ is a continuous function. The approach discussed below works also for $\sigma_{m i j} \neq 0$ under in-phase cycling if one considers $1 / n^{*}\left(\Delta \sigma, \sigma_{m}\right)$ as a continuous function of the two tensor variables, and the corresponding conditions on $\sigma_{m}$ as a function of coordinate and scaling parameter have to be applied as well.

If the material fatigue properties and/or stress field vary with the coordinate, one can write for a body $\Omega$ an (initial) durability condition under periodic cycling loading as

$$
n<\inf _{y \in \Omega} n^{*}(\Delta \sigma(y), y)
$$

where $n^{*}(\Delta \sigma(y), y)$ is the Wöhler diagram for a homogeneous material with the fatigue properties as at the point $y$, under the periodic cycling $\Delta \sigma_{i j}$ homogeneous in space coordinates.

Let us consider now a loading process with varying cycle parameters such that closed loops can be always identified but may be different. Let $m=1,2, \ldots$ be a number of a closed loop with the stress range $\Delta \sigma_{i j}(m, y)$ in the loading history $\{\sigma(\cdot, y)\}=\{\sigma(m, y)\}_{m=1}^{n}$ at the point $y$. Let $n^{*}(\Delta \sigma, y)$ be the Wöhler function for the material of the point $y$. The Palmgren-Miner linear damage accumulation rule gives the durability 
condition for a cycle $n$ in the form

$$
\omega^{N}(\{\sigma(\cdot, y)\} ; n, y):=\sum_{m=1}^{n} \frac{1}{n^{*}[\Delta \sigma(m, y), y]}<1 .
$$

where $\omega^{N}(\{\sigma(\cdot, y)\} ; n, y)$ is called fatigue damage measure.

The corresponding body durability condition has the the form

$$
\sup _{y \in \Omega} \omega^{N}(\{\sigma(\cdot, y)\} ; n, y)<1 .
$$

Local fatigue durability condition (3) is generally not applicable to singular stress fields. For more general classes of stress fields, e.g. $L_{2}(\Omega)$, a non-local fatigue durability condition can be applied. For example, for the periodic cycling we can take

$$
\begin{array}{r}
n<\inf _{y \in \Omega} n^{* \odot}(\Delta \sigma ; y), \quad n^{* \odot}(\Delta \sigma ; y):=n^{*}\left(\Delta \sigma^{\odot}(y), y\right), \\
\Delta \sigma_{i j}^{\odot}(y):=\int_{\Omega} w_{i j k l}(y, x) \Delta \sigma_{k l}(x) d x
\end{array}
$$

where $w(y, x)$ is as above. For the non-periodic cycling, one can replace $\Delta \sigma(n, y)$ by $\Delta \sigma^{\odot}(n, y)$ in the linear damage accumulation rule (4).

\section{ELEMENTS OF ASYMPTOTIC HOMOGENISATION}

A boundary value problem of elasticity for a composite solid $\Omega$ having an $\varepsilon Y$-periodic structure, that is a large number of periodically distributed inclusions or pores with a scaling parameter $\varepsilon$, is presented by displacement $u_{i}^{\varepsilon} \in H^{1}(\Omega)$ and stress $\sigma_{i j}^{\varepsilon} \in L^{2}(\Omega)$ fields for each $\varepsilon>0$. According to $[6,1,3]$, the homogenised displacement and stress fields, $u_{i}^{0} \in H^{1}(\Omega), \hat{\sigma}_{i j} \in L^{2}(\Omega)$, present a solution to a uniquely solvable homogenised problem of elasticity in the domain $\Omega$.

It is known $[1,3]$ that $\sigma_{i j}^{\varepsilon} \in L^{2}(\Omega)$ contains a subsequence, which two-scale converges to a function $\sigma^{0} \in L^{2}(\Omega \times Y)$, that is,

$$
\lim _{\varepsilon \rightarrow 0}\left|\int_{\Omega} \psi\left(x, \frac{x}{\varepsilon}\right) \sigma_{i j}^{\varepsilon}(x) d x-\frac{1}{|Y|} \int_{\Omega} \int_{Y} \psi(x, \xi) \sigma_{i j}^{0}(x, \xi) d x d \xi\right|=0,
$$

for any $\psi \in L^{2}\left(\Omega, C_{p e r}(Y)\right)$. Furthermore,

$$
\sigma_{i j}^{0}(x, \xi)=A_{i j k l}(\xi) \hat{\sigma}_{k l}(x),
$$

where $A_{i h j k}(\xi)$ is the stress concentration tensor [6, Chap.9, Sec.4], obtained after solution of an auxiliary periodic problem of elasticity and such that $\frac{1}{Y} \int_{Y} A_{i j k l}(\xi) d \xi=\delta_{i j} \delta_{k l}$. 


\section{HOMOGENISATION OF LOCAL STRENGTH AND DURABILITY CONDITIONS}

In a periodic medium, all becomes dependent on the scaling parameter $\varepsilon$. Strength condition (1) and fatigue durability condition (3) become

$$
\sup _{y \in \Omega} \Lambda^{\varepsilon}\left(\sigma^{\varepsilon}, y\right)<1, \quad n<\inf _{y \in \Omega} n^{* \varepsilon}\left(\Delta \sigma^{\varepsilon}, y\right) .
$$

Suppose,

$$
\Lambda^{\varepsilon}\left(\sigma^{\varepsilon}, y\right):=\Lambda\left(\sigma^{\varepsilon}, \frac{y}{\varepsilon}\right), \quad n^{* \varepsilon}\left(\Delta \sigma^{\varepsilon}, y\right):=n^{*}\left(\Delta \sigma^{\varepsilon}, \frac{y}{\varepsilon}\right) .
$$

Our aim is to derive macro-strength and macro-durability conditions like (1), (3) and (4)-(5), where the homogenised strength $\hat{\Lambda}$ function, Wöhler function $\hat{n}^{*}$, and damage measure $\hat{\omega}^{N}$ are functions of the homogenised stress $\hat{\sigma}_{i h}(x)$ and the composite micro-characteristics only. Let the notation $|\sigma|$ for a tensor $\sigma_{i j}$ mean a matrix norm.

Proposition 1 (homogenisation of local normalised equivalent strength function) Let a tensor function sequence $\sigma^{\varepsilon}(y) \in C(\bar{\Omega})$ converge to a tensor function $\sigma^{0}(y, \zeta) \in C\left(\bar{\Omega}, C_{\text {per }}(Y)\right)$ uniformly w.r.t. $y$ as $\varepsilon \rightarrow 0$, i.e.,

$$
\lim _{\varepsilon \rightarrow 0} \sup _{y \in \Omega}\left|\sigma^{\varepsilon}(y)-\sigma^{0}\left(y, \frac{y}{\varepsilon}\right)\right|=0
$$

and $\Lambda \in C\left(\mathbb{R}^{n \times n}, \mathcal{M}_{p e r}(Y)\right)$. Then,

$$
\lim _{\varepsilon \rightarrow 0} \sup _{y \in \Omega}\left|\Lambda\left(\sigma^{\varepsilon}(y), \frac{y}{\varepsilon}\right)-\Lambda\left(\sigma^{0}\left(y, \frac{y}{\varepsilon}\right), \frac{y}{\varepsilon}\right)\right|=0
$$

If $\sigma^{0}$ is expressed by (9), then

$\lim _{\varepsilon \rightarrow 0} \sup _{y \in \Omega} \Lambda\left(\sigma^{\varepsilon}(y), \frac{y}{\varepsilon}\right) \leq \sup _{y \in \Omega} \hat{\Lambda}(\hat{\sigma}(y)), \hat{\Lambda}(\hat{\sigma}(y)):=\sup _{\zeta \in Y} \Lambda\left(A_{i j k l}(\zeta) \hat{\sigma}_{k l}(y), \zeta\right)$.

and the limit sufficient local macro-strength condition is $\sup _{y \in \Omega} \hat{\Lambda}(\hat{\sigma}(y))<1$.

Proof:

$$
\begin{aligned}
& \sup _{y \in \Omega}\left|\Lambda\left(\sigma^{\varepsilon}(y), \frac{y}{\varepsilon}\right)-\Lambda\left(\sigma^{0}\left(y, \frac{y}{\varepsilon}\right), \frac{y}{\varepsilon}\right)\right| \leq \\
& \sup _{y \in \Omega} \sup _{\zeta \in Y}\left|\Lambda\left(\sigma^{\varepsilon}(y), \zeta\right)-\Lambda\left(\sigma^{0}\left(y, \frac{y}{\varepsilon}\right), \zeta\right)\right| .
\end{aligned}
$$

Let us take any $\delta_{1}>0$. Belonging $\Lambda(\sigma, \zeta)$ to $C\left(\mathbb{R}^{n \times n}, \mathcal{M}_{p e r}(Y)\right)$ implies that for any constant $C_{1}>0$ there exists $\delta_{2}>0$ such that $\sup _{\zeta \in Y} \mid \Lambda\left(\sigma^{\prime}, \zeta\right)-$ 
$\Lambda\left(\sigma^{\prime \prime}, \zeta\right) \mid<\delta_{1}$ for any tensors $\sigma^{\prime}, \sigma^{\prime \prime}$ such that $\left|\sigma^{\prime}\right|,\left|\sigma^{\prime \prime}\right| \leq C_{1}$ and $\left|\sigma^{\prime}-\sigma^{\prime \prime}\right|<\delta_{2}$. Due to (12) and belonging $\sigma^{0}(y, \zeta)$ to $C\left(\bar{\Omega}, C_{p e r}(Y)\right)$, one can choose such $\tilde{\varepsilon}>0$ that $\left|\sigma^{0}(y, \zeta)\right|,\left|\sigma^{\varepsilon}(y)\right| \leq C_{1}$ for some $C_{1}>0$ for any $\varepsilon<\tilde{\varepsilon}, y \in \bar{\Omega}$ and $\zeta \in \bar{Y}$. Let us take $\tilde{\varepsilon}>0$ even smaller such that $\left|\sigma^{\varepsilon}(y)-\sigma^{0}\left(y, \frac{y}{\varepsilon}\right)\right|<\delta_{2}$ due to (12). Thus, $\sup _{\zeta \in Y} \mid \Lambda\left(\sigma^{\varepsilon}(y), \zeta\right)-$ $\Lambda\left(\sigma^{0}\left(y, \frac{y}{\varepsilon}\right), \zeta\right) \mid<\delta_{1}$. This implies the convergency of the right hand side of (14) to zero as $\varepsilon \rightarrow 0$, that proves (13).

The rest of the proposition follows from (13), (9) and (10).

Note that although the hypotheses of Proposition 1 are satisfied for not any two-scale converging tensor function sequence $\sigma^{\varepsilon}(y) \in L^{2}(\Omega)$ appearing in the elastic composite analysis, the range of their validity is not empty. A trivial example is an infinite periodic composite with smooth inclusions, under a uniform load at infinity: then $\sigma^{\epsilon}(y)$ and $\sigma^{0}(y, y / \epsilon)$ simply coincide. Moreover, the hypotheses of Proposition 1 are always satisfied for the non-local counterpart of any two-scale converging tensor sequence, see Proposition 7 below.

Let us denote $n^{*-1}(\Delta \sigma, y):=1 / n^{*}(\Delta \sigma, y)$. Similarly to Proposition 1 , we have

Proposition 2 (homogenisation of local fatigue durability diagram) Let a periodic stress cycling have a tensor range sequence $\Delta \sigma^{\varepsilon}(y) \in$ $C(\bar{\Omega})$, which converges to a tensor function $\Delta \sigma^{0}(y, \zeta) \in C\left(\bar{\Omega}, C_{p e r}(Y)\right)$ uniformly w.r.t. $y$ as $\varepsilon \rightarrow 0$ i.e., $\lim _{\varepsilon \rightarrow 0} \sup _{y \in \Omega}\left|\Delta \sigma^{\varepsilon}(y)-\Delta \sigma^{0}\left(y, \frac{y}{\varepsilon}\right)\right|=0$. Let $n^{*}\left(\Delta \sigma, \frac{y}{\varepsilon}\right)$ be a durability diagram such that $n^{*-1} \in C\left(\mathbb{R}^{n \times n}, \mathcal{M}_{\text {per }}(Y)\right)$.

$$
\text { Then } \lim _{\varepsilon \rightarrow 0} \sup _{y \in \Omega}\left|n^{*-1}\left(\Delta \sigma^{\varepsilon}(y), \frac{y}{\varepsilon}\right)-n^{*-1}\left(\Delta \sigma^{0}\left(y, \frac{y}{\varepsilon}\right), \frac{y}{\varepsilon}\right)\right|=0 .
$$

If $\sigma^{0}$ is expressed by (9), then

$$
\begin{array}{cc} 
& \lim _{\varepsilon \rightarrow 0} \inf _{y \in \Omega} n^{*}\left(\Delta \sigma^{\varepsilon}(y), \frac{y}{\varepsilon}\right) \geq \inf _{y \in \Omega} \hat{n}^{*}(\Delta \hat{\sigma}(y)), \\
\text { where } & \hat{n}^{*}(\Delta \hat{\sigma}(y)):=\inf _{\zeta \in Y} n^{*}\left(A_{i j k l}(\zeta) \Delta \hat{\sigma}_{k l}(y), \zeta\right)
\end{array}
$$

and the limit sufficient local fatigue macro-durability condition under periodic cycling loading is $n<\inf _{y \in \Omega} \hat{n}^{*}(\hat{\sigma}(y))$.

Using Propositions 2 and the linear accumulation rule (4), (5), we can write the limiting expression for the Palmgren-Miner fatigue damage measure and the durability condition under varying cyclic loading.

Proposition 3 (homogenisation of local fatigue damage measure and durability condition) Let a stress cycling have a range $\Delta \sigma^{\varepsilon}(m, y)$ from $C(\bar{\Omega})$, which converges to a tensor function $\Delta \sigma^{0}(m, y, \zeta)$ 
from $C\left(\bar{\Omega}, C_{\text {per }}(Y)\right)$ uniformly w.r.t. y for each cycle number $m$, as $\varepsilon \rightarrow 0$, i.e., $\lim _{\varepsilon \rightarrow 0} \sup _{y \in \Omega}\left|\Delta \sigma^{\varepsilon}(m, y)-\Delta \sigma^{0}\left(m, y, \frac{y}{\varepsilon}\right)\right|=0$ for any $m$. Let $n^{*}\left(\Delta \sigma, \frac{y}{\varepsilon}\right)$ be a durability diagram such that $n^{*-1} \in C\left(\mathbb{R}^{n \times n}, \mathcal{M}_{\text {per }}(Y)\right)$. Let $\omega^{N}\left(\left\{\sigma^{\varepsilon}(\cdot, y)\right\} ; n, \frac{y}{\varepsilon}\right)=\sum_{m=1}^{n} n^{*-1}\left(\Delta \sigma^{\varepsilon}(m, y), \frac{y}{\varepsilon}\right)$. Then

$$
\lim _{\varepsilon \rightarrow 0} \sup _{y \in \Omega}\left|\omega^{N}\left(\left\{\sigma^{\varepsilon}(\cdot, y)\right\} ; n, \frac{y}{\varepsilon}\right)-\omega^{N}\left(\left\{\sigma^{0}\left(\cdot, y, \frac{y}{\varepsilon}\right)\right\} ; n, \frac{y}{\varepsilon}\right)\right|=0,
$$

If $\sigma^{0}$ is expressed by (9), then

$$
\begin{array}{r}
\lim _{\varepsilon \rightarrow 0} \sup _{y \in \Omega} \omega^{N}\left(\left\{\sigma^{\varepsilon}(\cdot, y)\right\} ; n, \frac{y}{\varepsilon}\right) \leq \sup _{y \in \Omega} \hat{\omega}^{N}(\{\hat{\sigma}(\cdot, y)\} ; n), \quad \text { where } \\
\hat{\omega}^{N}(\{\hat{\sigma}(\cdot, y)\} ; n):=\sup _{\zeta \in Y} \omega^{N}\left(\left\{A_{i j k l}(\zeta) \hat{\sigma}_{k l}(\cdot, y)\right\} ; n, \zeta\right)= \\
\sup _{\zeta \in Y} \sum_{m=1}^{n} \frac{1}{n^{*}\left(A_{i j k l}(\zeta) \Delta \hat{\sigma}_{k l}(m, y), \zeta\right)} \leq \sum_{m=1}^{n} \frac{1}{\hat{n}^{*}(\Delta \hat{\sigma}(m, y))}
\end{array}
$$

and the limit sufficient local fatigue macro-durability condition under variable cyclic loading is

$$
\sup _{y \in \Omega} \hat{\omega}^{N}(\{\hat{\sigma}(\cdot, y)\} ; n)<1 .
$$

Proof: Using (15), we have

$$
\begin{aligned}
\lim _{\varepsilon \rightarrow 0} \sup _{y \in \Omega}\left|\omega^{N}\left(\left\{\sigma^{\varepsilon}(\cdot, y)\right\} ; n, y\right)-\omega^{N}\left(\left\{\sigma^{0}\left(\cdot, y, \frac{y}{\varepsilon}\right)\right\} ; n, y\right)\right| & \leq \\
\sum_{m=1}^{n} \lim _{\varepsilon \rightarrow 0} \sup _{y \in \Omega}\left|n^{*-1}\left(\Delta \sigma^{\varepsilon}(m, y), \frac{y}{\varepsilon}\right)-n^{*-1}\left(\Delta \sigma^{0}(m, y,), \frac{y}{\varepsilon}\right)\right| & =0 .
\end{aligned}
$$

This proves (16), and the rest of the proposition does directly follow.

Note that according to (17), the limit composite damage measure is generally not expressed but only estimated by the damage measure based on the limit composite durability diagram.

\section{HOMOGENISATION OF NON-LOCAL STRENGTH AND DURABILITY CONDITIONS}

Let us consider for a periodic medium the limits of non-local microstrength and micro-durability conditions $\sup _{y \in \Omega} \Lambda^{\odot \varepsilon}\left(\sigma^{\varepsilon} ; y\right)<1, \quad n<$ $\inf _{y \in \Omega} n^{* \odot \varepsilon}\left(\Delta \sigma^{\varepsilon} ; y\right)$ as $\varepsilon \rightarrow 0$. Representations (2), (6) become

$$
\begin{gathered}
\Lambda^{\odot \varepsilon}\left(\sigma^{\varepsilon} ; y\right):=\Lambda^{\varepsilon}\left(\sigma^{\odot \varepsilon}(y), y\right), \quad n^{* \odot \varepsilon}\left(\Delta \sigma^{\varepsilon} ; y\right):=n^{* \varepsilon}\left(\Delta \sigma^{\odot \varepsilon}(y), y\right), \\
\sigma_{i j}^{\odot \varepsilon}(y):=\int_{\Omega} w_{i j k l}^{\varepsilon}(y, x) \sigma_{k l}^{\varepsilon}(x) d x, \quad y \in \Omega .
\end{gathered}
$$


Suppose the functions $\Lambda^{\varepsilon}$ and $n^{* \varepsilon}$ have form (11). Let further $w_{i j k l}^{\varepsilon}(y, x):=w_{i j k l}\left(y, \frac{y}{\varepsilon}, x, \frac{x}{\varepsilon}\right)$. Then

$$
\begin{aligned}
& \Lambda^{\varepsilon}\left(\sigma^{\odot \varepsilon}, y\right)=\Lambda\left(\sigma^{\odot \varepsilon}(y), \frac{y}{\varepsilon}\right), n^{* \varepsilon}\left(\Delta \sigma^{\odot \varepsilon}, y\right)=n^{*}\left(\Delta \sigma^{\odot \varepsilon}(y), \frac{y}{\varepsilon}\right), \\
& \sigma_{i j}^{\odot \varepsilon}(y)=\int_{\Omega} w_{i j k l}\left(y, \frac{y}{\varepsilon}, x, \frac{x}{\varepsilon}\right) \sigma_{k l}^{\varepsilon}(x) d x,
\end{aligned}
$$

Lemma 4 Let $\sigma^{\varepsilon} \in L^{2}(\Omega)$ be a sequence of tensor functions $\sigma^{\varepsilon}(x)$ twoscale converging to a tensor $\sigma^{0} \in L^{2}(\Omega \times Y)$ as $\varepsilon \rightarrow 0$. Let $w \in$ $C\left(\bar{\Omega}, C_{p e r}\left(Y, L^{2}\left(\Omega, C_{p e r}(Y)\right)\right)\right)$. Then the sequence

$$
\tilde{\sigma}^{\odot \varepsilon}(y, \zeta):=\int_{\Omega} w\left(y, \zeta, x, \frac{x}{\varepsilon}\right) \sigma^{\varepsilon}(x) d x,
$$

is bounded in $C\left(\bar{\Omega}, C_{\text {per }}(Y)\right)$ and does strongly converge in this space to

$$
\sigma^{\odot 0}(y, \zeta)=\frac{1}{|Y|} \int_{\Omega} \int_{Y} w(y, \zeta, x, \xi) \sigma^{0}(x, \xi) d \xi d x .
$$

Proof: Since the sequence $\sigma^{\varepsilon} \in L^{2}(\Omega)$ two-scale converges, it converges also weakly and, consequently is bounded in $L^{2}(\Omega)$, that is, $\left\|\sigma^{\varepsilon}\right\|_{L^{2}(\Omega)}<$ $C<\infty$.

The periodicity in $\zeta$ is evident for the both functions $\tilde{\sigma}^{\odot \varepsilon}(y, \zeta)$ and $\sigma^{\odot 0}(y, \zeta)$, and it is sufficient to prove the proposition in the space $C(\bar{\Omega} \times$ $\bar{Y})$.

From (19) we have,

$$
\sup _{y \in \bar{\Omega}} \sup _{\zeta \in \bar{Y}}\left|\tilde{\sigma}^{\odot \varepsilon}(y, \zeta)\right| \leq \sup _{y \in \bar{\Omega}} \sup _{\zeta \in \bar{Y}}\left[\int_{\Omega}\left|w\left(y, \zeta, x, \frac{x}{\varepsilon}\right)\right|^{2} d x\right]^{\frac{1}{2}}\left\|\sigma^{\varepsilon}\right\|_{L^{2}(\bar{\Omega})} \leq
$$

$\sup _{y \in \bar{\Omega}} \sup _{\zeta \in \bar{Y}}\left[\int_{\Omega} \sup _{\xi \in Y}|w(y, \zeta, x, \xi)|^{2} d x\right]^{\frac{1}{2}} C=\|w\|_{C\left(\bar{\Omega}, C_{p e r}\left(Y, L^{2}\left(\Omega, C_{p e r}(Y)\right)\right)\right)} C$.

That is, the sequence $\tilde{\sigma}^{\odot \varepsilon}(y, \zeta)$ is equi-bounded in $C(\bar{\Omega} \times \bar{Y})$. Let us check the continuity,

$$
\begin{aligned}
& \left|\tilde{\sigma}^{\odot \varepsilon}\left(y_{1}, \zeta_{1}\right)-\tilde{\sigma}^{\odot \varepsilon}\left(y_{2}, \zeta_{2}\right)\right|= \\
& \left|\int_{\Omega}\left[w\left(y_{1}, \zeta_{1}, x, \frac{x}{\varepsilon}\right)-w\left(y_{2}, \zeta_{2}, x, \frac{x}{\varepsilon}\right)\right] \sigma^{\varepsilon}(x) d x\right| \leq \\
& {\left[\int_{\Omega} \sup _{\xi \in Y}\left|w\left(y_{1}, \zeta_{1}, x, \xi\right)-w\left(y_{2}, \zeta_{2}, x, \xi\right)\right|^{2} d x\right]^{\frac{1}{2}}\left\|\sigma^{\varepsilon}\right\|_{L^{2}(\bar{\Omega})}=} \\
& \left\|w\left(y_{1}, \zeta_{1}, \cdot, \cdot\right)-w\left(y_{2}, \zeta_{2}, \cdot, \cdot\right)\right\|_{L^{2}\left(\Omega, C_{p e r}(Y)\right)} C .
\end{aligned}
$$


The term $\left\|w\left(y_{1}, \zeta_{1}, \cdot, \cdot\right)-w\left(y_{2}, \zeta_{2}, \cdot, \cdot\right)\right\|_{L^{2}\left(\Omega, C_{p e r}(Y)\right)}$ tends to zero as $\sqrt{\left|y_{1}-y_{2}\right|^{2}+\left|\zeta_{1}-\zeta_{2}\right|^{2}} \rightarrow 0$ uniformly w.r.t. $\left\{y_{i}, \zeta_{i}\right\} \in \bar{\Omega} \times \bar{Y}, i=1,2$, since $w \in C\left(\bar{\Omega}, C_{\text {per }}\left(Y, L^{2}\left(\Omega, C_{p e r}(Y)\right)\right)\right)$. Thus, the sequence $\tilde{\sigma}^{\odot \varepsilon}(y, \zeta)$ does not only belong to $C(\bar{\Omega} \times \bar{Y})$ but is also equi-bounded and equicontinuous. From the Ascoli-Arzelá theorem, the sequence is then compact in $C(\bar{\Omega} \times \bar{Y})$.

On the other hand, as follows from the two-scale convergency of $\sigma^{\varepsilon}$, the sequence $\tilde{\sigma}^{\odot \varepsilon}(y, \zeta)$ converges point-wise to $\sigma^{\odot 0}(y, \zeta)$ for any $\{y, \zeta\} \in \bar{\Omega} \times$ $\bar{Y}$. Since the sequence is also equi-bounded, this means it converges to $\sigma^{\odot 0}(y, \zeta)$ weakly in $C(\bar{\Omega} \times \bar{Y})$ (see, e.g., [4]). However, each compact and weakly converging sequence in a Banach space converges also strongly (see, e.g., [7, Section 20.2]). This proves the lemma.

Proposition 5 Let $\sigma^{\varepsilon} \in L^{2}(\Omega)$ be a sequence of tensor functions $\sigma^{\varepsilon}(x)$ two-scale converging to a tensor $\sigma^{0} \in L^{2}(\Omega \times Y)$. Suppose, $w \in$ $C\left(\bar{\Omega}, C_{\text {per }}\left(Y, L^{2}\left(\Omega, C_{\text {per }}(Y)\right)\right)\right)$. Then the sequence $\sigma^{\odot \varepsilon}(y)$ given by (18) is bounded in $C(\bar{\Omega})$ and does converge to the tensor function $\sigma^{\odot 0}\left(y, \frac{y}{\varepsilon}\right)$, given by (20), uniformly w.r.t. y as $\varepsilon \rightarrow 0$, i.e.,

$$
\lim _{\varepsilon \rightarrow 0} \sup _{y \in \bar{\Omega}}\left|\sigma^{\odot \varepsilon}(y)-\sigma^{\odot 0}\left(y, \frac{y}{\varepsilon}\right)\right|=0 .
$$

Proof: Let us note that $\sigma^{\odot \varepsilon}(y)=\tilde{\sigma}^{\odot \varepsilon}\left(y, \frac{y}{\varepsilon}\right)$, where the sequence $\tilde{\sigma}^{\odot \varepsilon}$ is given by (19) and belongs to and is equi-bounded in $C\left(\bar{\Omega}, C_{p e r}(Y)\right)$ according to Lemma 4. This implies the sequence $\sigma^{\odot \varepsilon}(y)$ belongs to and is equi-bounded in $C(\bar{\Omega})$. Then, owing to Proposition 4 ,

$\sup _{y \in \bar{\Omega}}\left|\sigma^{\odot \varepsilon}(y)-\sigma^{\odot 0}\left(y, \frac{y}{\varepsilon}\right)\right|=\sup _{y \in \bar{\Omega}}\left|\tilde{\sigma}^{\odot \varepsilon}\left(y, \frac{y}{\varepsilon}\right)-\sigma^{\odot 0}\left(y, \frac{y}{\varepsilon}\right)\right| \leq$ $\sup _{y \in \bar{\Omega}} \sup _{\zeta \in \bar{Y}}\left|\tilde{\sigma}^{\odot \varepsilon}(y, \zeta)-\sigma^{\odot 0}(y, \zeta)\right| \rightarrow 0$.

Applying Proposition 1 to the non-local stresses (20) and taking into account their convergency proved in Proposition 5, we arrive at the following proposition on homogenisation of non-local strength conditions.

Proposition 6 Let $\sigma^{\varepsilon} \in L^{2}(\Omega)$ be a sequence of tensor functions twoscale converging to a tensor $\sigma^{0} \in L^{2}(\Omega \times Y)$. Suppose the non-local stress $\sigma^{\odot \varepsilon}(y)$ is given by (18) with the weight $w \in C\left(\bar{\Omega}, C_{\text {per }}\left(Y, L^{2}\left(\Omega, C_{p e r}(Y)\right)\right)\right)$. Suppose $\Lambda \in C\left(\mathbb{R}^{n \times n}, \mathcal{M}_{\text {per }}(Y)\right)$. Then

$$
\lim _{\varepsilon \rightarrow 0} \sup _{y \in \Omega}\left|\Lambda\left(\sigma^{\odot \varepsilon}(y), \frac{y}{\varepsilon}\right)-\Lambda\left(\sigma^{\odot 0}\left(y, \frac{y}{\varepsilon}\right), \frac{y}{\varepsilon}\right)\right|=0,
$$

where $\sigma^{\odot 0}(y, \zeta)$ is given by (20). If $\sigma^{0}$ is expressed by (9), then

$$
\lim _{\varepsilon \rightarrow 0} \sup _{y \in \Omega} \Lambda\left(\sigma^{\odot \varepsilon}(y), \frac{y}{\varepsilon}\right) \leq \sup _{y \in \Omega} \hat{\Lambda} \odot(\hat{\sigma} ; y)
$$




$$
\begin{aligned}
\text { where } \quad & \hat{\Lambda} \odot(\hat{\sigma} ; y):=\sup _{\zeta \in Y} \Lambda\left(\int_{\Omega} \hat{w}_{i j k l}(y, \zeta, x) \hat{\sigma}_{k l}(x) d x, \zeta\right) \\
& \hat{w}_{i j k l}(y, \zeta, x)=\frac{1}{|Y|} \int_{Y} w_{i j p q}(y, \zeta, x, \xi) A_{p q k l}(\xi) d \xi
\end{aligned}
$$

Finally the limit sufficient non-local macro-strength condition is $\sup _{y \in \Omega} \hat{\Lambda}^{\odot}(\hat{\sigma} ; y)<1$.

Changing the notations, we obtain a similar proposition on homogenisation of non-local fatigue durability diagram $n^{*}$ and then of the fatigue damage measure $\omega$.

The approach developed in the paper is based on the two-scale convergence for stresses (8) following from [1], see also [3], for the linear elasticity. It will also work for more complex material behaviour, e.g. plasticity or small-cyclic fatigue if the convergence (8) holds true for such cases.

\section{Acknowledgments}

This work was completed under the research grant GR/M24592 "Non-local approach to high cyclic fatigue: Theoretical basis" of the Engineering and Physical Sciences Research Council, UK.

\section{References}

[1] Allaire, G (1992) Homogenisation and Two-Scale Convergence, SIAM J. Math. Anal., 23 , 1482-1518.

[2] Bakhvalov, N and Panasenko, G (1984) Homogenisation: Averaging Processes in Periodic Media. Mathematical Problems in the Mechanics of Composite Materials, Dordrecht-Boston-London:Kluwer.

[3] Cioranescu, D and Donato, P (1999) An Introduction to Homogenization. New York: Oxford University Press.

[4] Kolmogorov, A N and Fomin, S B (1957) Elements of the theory of functions and functional analysis. Rochester, N.Y.: Graylock Press.

[5] Mikhailov, S E (1995) A Functional Approach to Non-local Strength Conditions and Fracture Criteria: I. Body and Point Fracture, Eng. Fract. Mech., 52, 731-743.

[6] Pobedrya, B E (1984) Mechanics of Composite Materials, Moscow State University Publishing.

[7] Trenogin, V A (1980) Functional Analysis, Moscow: Nauka. 